\title{
Uromyces trifolii, a new addition to rust fungi of Himachal Pradesh, India, with a checklist of Uromyces in India
}

\author{
Gautam $\mathrm{AK}^{1^{*}}$ and Avasthi $\mathrm{S}^{2}$ \\ ${ }^{1}$ School of Agriculture, Faculty of Science, Abhilashi University, Mandi (H.P.) 175028, India \\ ${ }^{2}$ Department of Botany, Abhilashi Institute of Life Sciences, Mandi (H.P.) 175008, India
}

Gautam AK, Avasthi S 2017 - Uromyces trifolii, a new addition to rust fungi of Himachal Pradesh, India with a checklist of Uromyces in India. Plant Pathology \& Quarantine 7(1), 1-14, Doi $10.5943 / \mathrm{ppq} / 7 / 1 / 1$

\begin{abstract}
Uromyces is a genus of rust fungi that infects both monocots and dicots throughout the world. The genus is particularly common on plant families like Asteraceae, Euphorbiaceae, Fabaceae, Liliaceae, Poaceae, and Loranthaceae. A rust infection was observed on leaves and stem of Trifolium repens from Himachal Pradesh, India. The symptoms appeared as dark brown to blackish brown pustules. Morphological and microscopic analyses of diseased samples identified it as Uromyces trifolii, which is new to Himachal Pradesh. Taxonomic descriptions and illustrations of the specimen are given. A checklist to assess diversity and distribution of the genus Uromyces in India is provided.
\end{abstract}

Key words - checklist - Himachal Pradesh - new record - rust fungi - Trifolium repens

\section{Introduction}

Uromyces (Link) Unger, a genus of rust fungi was proposed by Unger (1833). There are more than 1562 taxonomic names within this genus worldwide (IndexFungorum 2016). According to Cummins \& Hiratsuka (2003) it is the second largest genus of rust fungi next to Puccinia and contains more than 600 reported species. It is mainly characterized by 1-celled teliospores which differentiate it from Puccinia, which has 2-celled teliospores. Uromyces infects both monocots and dicots throughout the world, infecting plant families like Asteraceae, Euphorbiaceae, Fabaceae, Liliaceae, and Poaceae, but Loranthaceae is a major one (Vidal-Russell \& Nickrent 2008).

The genus has a wide diversity and host range in India also. Himachal Pradesh, the northern hilly state of India is situated in the western part of Himalaya. It is a mountainous state with elevations ranging from about 350-7,000 $\mathrm{m}$ above sea level. Climatic conditions vary from hot and sub-humid tropical in the southern tracts to cold, alpine and glacial in the northern and eastern mountain ranges. The changeable geographical and climatic conditions of the state are favourable for biodiversity including growth and development of plant pathogens. One of the most important characteristics of plant rusts is their exceptionally high degree of host specificity. The vast biodiversity and climatic conditions of the state lead to a wide rust diversity, distribution and host range of this fungal group. 
A rust infection was observed on leaves and stems of Trifolium repen L. (Fabaceae) during our routine phytopathological survey from district Mandi of Himachal Pradesh in 2015. A detailed taxonomic study and survey of the literature as well as comparative analyses revealed that the fungus is a new addition to the rust fungi in Himachal Pradesh (Mukherji \& Juneja 1974, Sarbhoy 1975, 1980, Bilgrami 1991, Jamaluddin et al. 2004). The disease was studied further and is described in the present study. A checklist to assess diversity and distribution of the genus Uromyces in India is also provided.

\section{Materials and methods}

Naturally infected plant parts showing rust symptoms were collected from Chail Chowk area of district Mandi, Himachal Pradesh. Infected leaves and stems were placed in separate polythene bags and taken to the laboratory for further examination. Specimens are deposited in the Abhilashi University Mycological Herbarium (AUMH), School of Agriculture, Faculty of Science, Abhilashi University, Mandi, Himachal Pradesh, India. Morphological features of all specimens were noted, and measurements were made. Diseased spots were photographed with a Sony DSC-X80 camera. Mounts of spores and free-hand sections of sori were prepared in lactophenol and heated to boiling. The fungal structures were examined under a light microscope (Olympus). At least 25 measurements for each microscopic structure were taken. Taxonomic analyses were made by consulting relevant literature and illustrations are given in accordance with Cummins \& Hiratsuka (2003).

A checklist of the genus Uromyces was also prepared to assess diversity and distribution in India. An exhaustive bibliographic survey of the literature published in various national and international journals, monographs, books, book chapters and magazines on these rust fungi was carried out. Some species names as reported in the cited publications have been replaced with their currently accepted name according to the Species Fungorum website (Speciesfungorum 2016).

\section{Results}

Trifolium repens is a perennial, herbaceous, low growing flowering plant of family Fabaceae and is commonly known as white clover. It is considered as a folk medicine in India, used against intestinal helminthic worms. An experimental in vivo study validated that the aerial shoots of $T$. repens bear significant anticestodal properties (Yadav et al. 2004). On leaves and stem dark brown to blackish brown telial pustules were observed (Fig. 1).

\section{Taxonomy}

Uromyces trifolii (R. Hedw.) Lév., Annls Sci. Nat., Bot., sér. 3, 8: 371 (1847)

(Figs. 1, 2)

$=$ Uromyces flectens Lagerh., Svensk bot. Tidskr. 3: 36 (1909).

$=$ Uromyces nerviphilus (Grognot) Hotson, Publ. Puget Sound Biol. Sta. Univ. Wash. 4: 368 (1925).

Telia mostly hypophyllous, sometimes epiphyllous, rounded, scattered, subepidermal, erumpent, pulverulent, surrounded by the ruptured epidermis, dark brown to blackish brown, 0.08 $0.19 \times 0.09-0.3 \mathrm{~mm}$. Teliospores globose to subglobose or ellipsoid to obovoid, $21-30 \times 15-24$ $\mu \mathrm{m}$ (mean $26.0 \times 19.6 \mu \mathrm{m}$ ), the apex rounded with minute hyaline papilla; wall $1-2 \mu \mathrm{m}$ thick, brown to chestnut brown, smooth or with minute scattered warts; apex 3-4 $\mu$ m thick, germ pore 1; pedicel hyaline, $4-8 \mu \mathrm{m}$ wide and up to $24 \mu \mathrm{m}$ long. Spermagonia, aecia and uredinia not found.

Material examined - On Trifolium repens L., with III stage, India, Himachal Pradesh, Chail Chowk (Mandi), at 1400 m, 24 August 2015, coll. Ajay K. Gautam (AUMH 1030). 


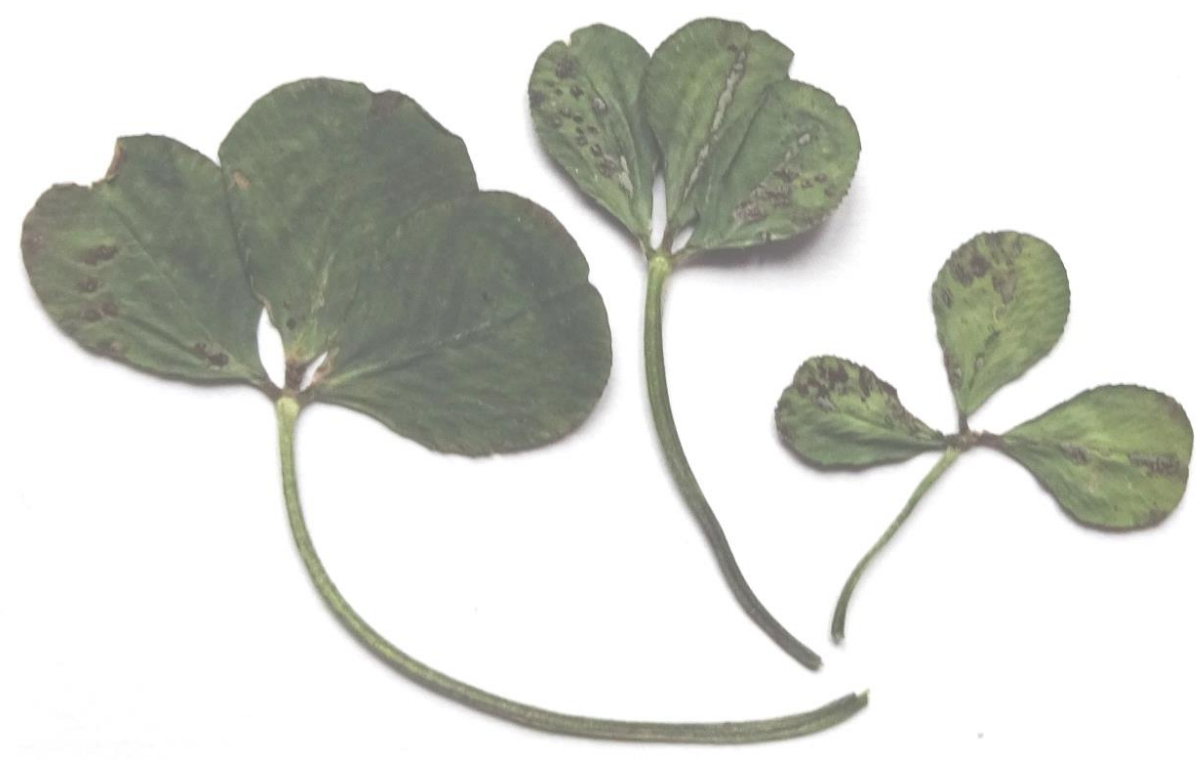

Fig 1 - Rust infection on Trifolium repens caused by Uromyces trifolii.

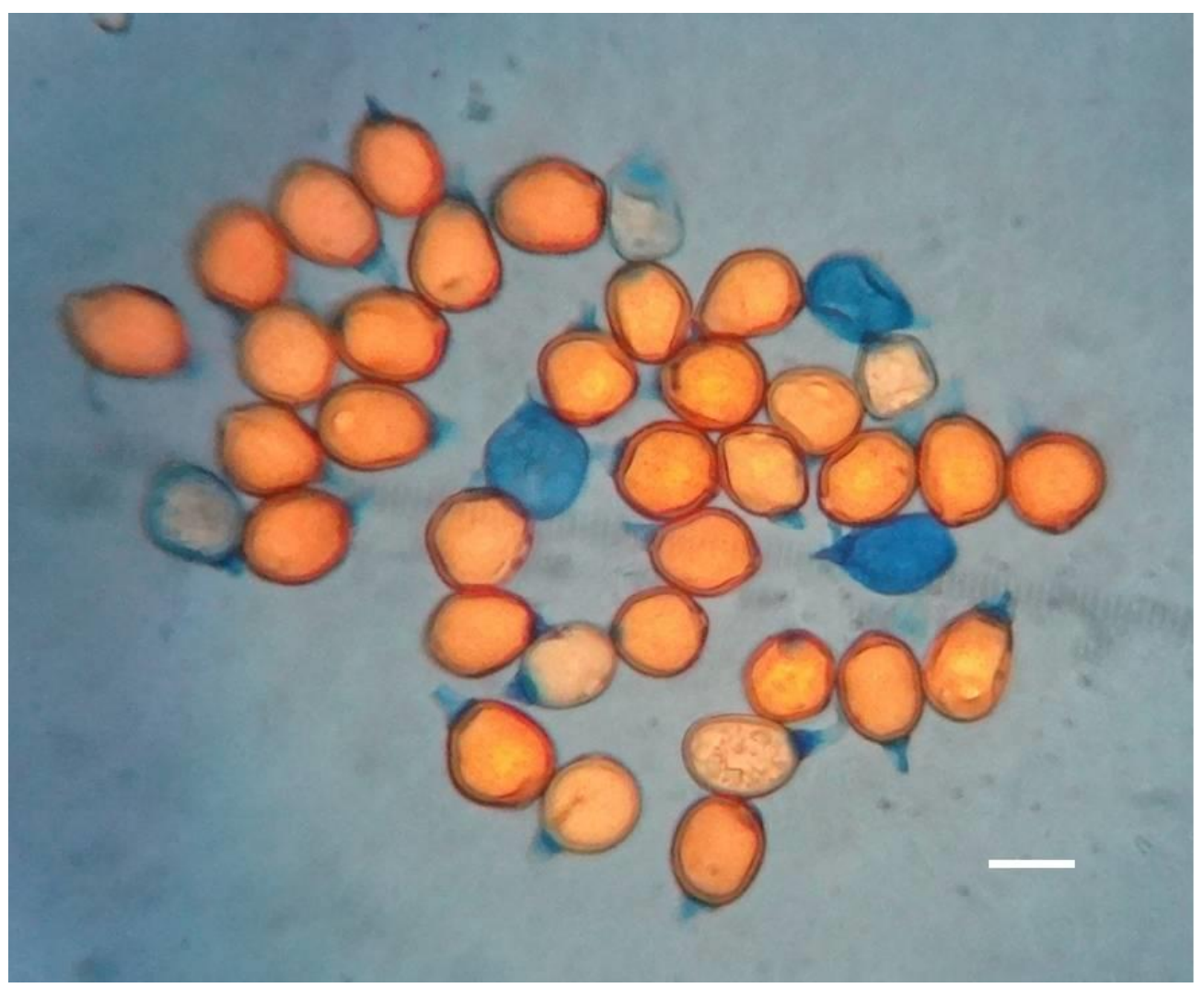

Fig 2 - Uromyces trifolii: Teliospores. Scale bar $=20 \mu \mathrm{m}$.

\section{Diversity and distribution}

Species of Uromyces infect both monocots and dicots throughout the world. Ninety-seven species of Uromyces have been identified and reported from India on 180 plant host species that belongs to 85 genera and 32 families. Thirty species have been found associated with family Fabaceae followed by Poaceae (23), Asteraceae (6), Lamiaceae and Caprifoliaceae (3 each) and Polygonaceae, Cyperaceae, Euphorbiaceae, Asparagaceae, Acanthaceae and Oleaceae (2 each). 
The remaining host plant families are associated with a single species of Uromyces (Table 1). Of 97 Uromyces species reported from India, only 15 species are reported from Himachal Pradesh. Uromyces species have also been found in nineteen other states of India (Table 1).

\section{Discussion}

Based on morphological characteristics the rust on Trifolium repens was identified as Uromyces trifolii. This rust has been previously reported on T. resupinatum (reversed clover) and T. pratense (red clover) from Haryana and Uttrakhand, respectively (Sydow \& Butler 1907a, b, Padwick \& Khan 1944, Hooda \& Saini 1990, Sokhi et al. 1985).

The genus Uromyces is mainly characterized by 1-celled teliospores. The Fabaceae (legume, pea, or bean family) and Poaceae (grass family) are particularly susceptible to infection of Uromyces spp. In India, 30 species of Uromyces have been reported on legumes and 23 species on grasses. Nearly 180 plant species that belong to 85 genera and 32 families are infected with Uromyces spp.

Table 1 Uromyces species recorded in India.

\begin{tabular}{|c|c|c|c|}
\hline Taxa & Hosts & Distribution* & References \\
\hline $\begin{array}{l}\text { U. pavgei Goswami } \\
\text { \& Nagachan }\end{array}$ & Achyranthes aspera & $\mathrm{AS}$ & Goswami \& Nagachan 1979 \\
\hline $\begin{array}{l}\text { U. lycoctoni } \\
\text { (Kalchbr.) Fuckel }\end{array}$ & Aconitum leave & $\mathrm{J} \& \mathrm{~K}$ & Arthur 1934, Cummins 1943 \\
\hline U. aconiti Fuckel & Aconitum lycoctonum & $\mathrm{J} \& \mathrm{~K}$ & $\begin{array}{l}\text { Arthur 1934, Arthur \& } \\
\text { Cummins } 1936\end{array}$ \\
\hline $\begin{array}{l}\text { U. acori T.S. } \\
\text { Ramakr. \& } \\
\text { Rangaswamy }\end{array}$ & Acorus calamus & $\mathrm{TN}$ & $\begin{array}{l}\text { Ramakrisnan \& Rangaswamy } \\
1948\end{array}$ \\
\hline $\begin{array}{l}\text { U. sporogoni } \\
\text { subsp. asiaticus } \\
\text { (T.S. Ramakr. \& } \\
\text { Rangaswami) } \\
\text { Parmelee \& Savile }\end{array}$ & Acorus calamus & $\mathrm{TN}$ & $\begin{array}{l}\text { Ramakrisnan \& Sundaram } \\
\text { 1955a }\end{array}$ \\
\hline $\begin{array}{l}\text { U. agropyri } \\
\text { Barclay }\end{array}$ & Agropyron sp. & $\mathrm{HP}$ & Barclay 1891 \\
\hline $\begin{array}{l}\text { U. sphaeropleus } \\
\text { Cooke }\end{array}$ & Allium сера & MS & Cooke 1876 \\
\hline $\begin{array}{l}\text { U. aloes (Cooke) } \\
\text { Magnus }\end{array}$ & $\begin{array}{l}\text { Aloe spicata, } \\
\text { Aloe vera }\end{array}$ & TN, MS & Ajrekar \& Tonapy 1923 \\
\hline $\begin{array}{l}\text { U. apludae Syd. \& } \\
\text { E.J. Butler }\end{array}$ & Alpuda aristata & MS, PB & Sydow \& Butler 1907a \\
\hline $\begin{array}{l}\text { U. amphilophis- } \\
\text { insculptae T.S. } \\
\text { Ramakr., Sriniv. \& } \\
\text { Sundaram }\end{array}$ & Amphilophus insculpta & $\mathrm{TN}$ & Ramakrisnan 1952 \\
\hline \multirow{2}{*}{$\begin{array}{l}\text { U. andropogonis- } \\
\text { annulati Syd., P. } \\
\text { Syd. \& E.J. Butler }\end{array}$} & Andropogon annulatus & $\begin{array}{l}\text { BR, UP, UK, } \\
\text { AP, MS, MP }\end{array}$ & Sydow \& Butler 1907a, b \\
\hline & Andropogon pertussis & $\mathrm{UP}, \mathrm{TN}$ & Ramakrisnan 1951a \\
\hline
\end{tabular}




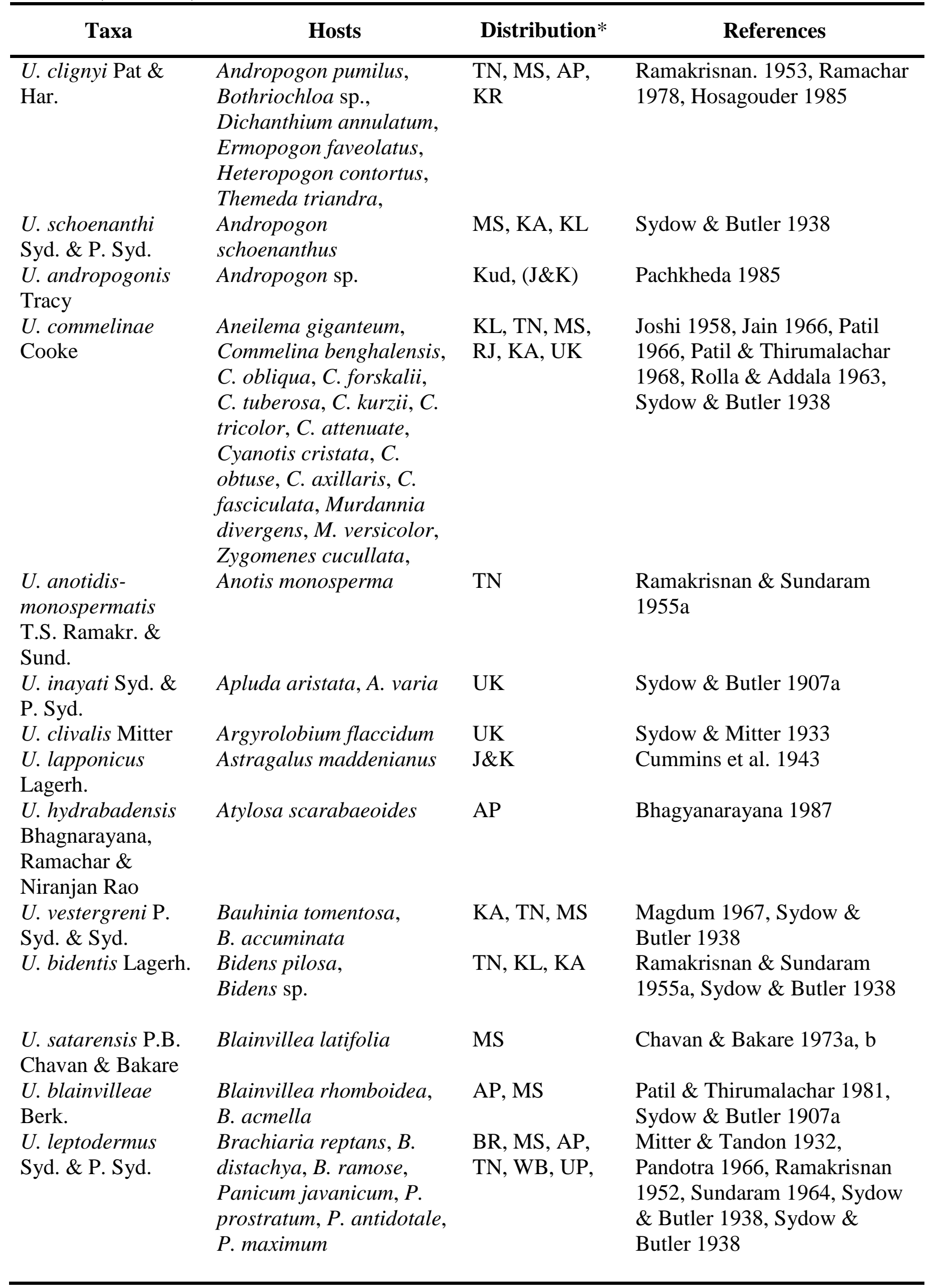


Table 1 (continued)

\begin{tabular}{|c|c|c|c|}
\hline Taxa & Hosts & Distribution* & References \\
\hline U. ambiens Cooke & Buxus sempervirens & $\mathrm{UK}, \mathrm{HP}$ & $\begin{array}{l}\text { Barclay 1891, Cooke 1874, } \\
\text { Sydow } 1913\end{array}$ \\
\hline $\begin{array}{l}\text { U. loculiformis T.S. } \\
\text { Ramakr. \& K. } \\
\text { Ramakr. }\end{array}$ & Chlorophytum attenuatum & $\mathrm{TN}$ & $\begin{array}{l}\text { Ramakrisnan \& Ramakrisnan } \\
1948\end{array}$ \\
\hline $\begin{array}{l}\text { U. ciceris-arietini } \\
\text { (Grognot.) Jacz. \& } \\
\text { G. Boyer }\end{array}$ & $\begin{array}{l}\text { Cicer arietinum, } \\
\text { Lathyrus aphaca, } \\
\text { L. odoratus, } \\
\text { Trogonella corniculata, } T \text {. } \\
\text { polyrata, Vicia biennis, } V . \\
\text { ervillia, } V . \text { faba, } V . \\
\text { gracilis, } V . \text { hirsuta, } V . \\
\text { narbonensis, } V . \text { sativa, } V . \\
\text { tetrasperma }\end{array}$ & $\begin{array}{l}\text { BR, MS, MP, } \\
\text { UP, DL, HP }\end{array}$ & $\begin{array}{l}\text { Agarwal 1959, Asthana 1957, } \\
\text { Bhahadur \& Singh 1967, } \\
\text { Butler \& Mcrae 1930, Jain } \\
\text { 1966, Payak 1949, Payak } \\
\text { 1962, Vasudeva } 1950\end{array}$ \\
\hline $\begin{array}{l}\text { U. nilagiricus T.S. } \\
\text { Ramakr. \& K. } \\
\text { Ramakr. }\end{array}$ & $\begin{array}{l}\text { Citrus reticulata, } \\
\text { Dendrophthoe falcata, } \\
\text { Loranthus sp., }\end{array}$ & $\mathrm{TN}, \mathrm{MS}$ & Chavan 1975, Ponappa 1961 \\
\hline U. decorates Syd. & $\begin{array}{l}\text { Crotalaria juncea, } \\
\text { C.rustica, C. retusa, C. } \\
\text { medicagenia }\end{array}$ & $\begin{array}{l}\text { AP, UK, KR, } \\
\mathrm{UP}, \mathrm{MS}, \mathrm{KL}, \\
\mathrm{BR}\end{array}$ & $\begin{array}{l}\text { Behera \& Mukherji 1974, } \\
\text { Chaudhari \& Singh 1974, } \\
\text { Hosagouder 1985, Khan 1994, } \\
\text { Ramakrisnan \& Sundaram } \\
\text { 1955a, Patil 1966, Payak 1949, } \\
\text { Prakash. } 1979\end{array}$ \\
\hline $\begin{array}{l}\text { Uromyces } \\
\text { achrous Syd. \& P. } \\
\text { Syd. }\end{array}$ & Dalbergia latifolia & KL & Hosagouder 1985 \\
\hline U. ascorus Syd. & Dalbergia latifolia & KL & Hosagouder 1985 \\
\hline $\begin{array}{l}\text { U. capitatus Syd. \& } \\
\text { P. Syd. }\end{array}$ & Desmodium tiliaefolia & UK & Sydow 1913, Sydow 1937 \\
\hline & Dicanthium annulatum & MP & Mishra \& Nema 1976 \\
\hline $\begin{array}{l}\text { U. appendiculatus } \\
\text { (Pers.) Link }\end{array}$ & $\begin{array}{l}\text { Dolichos lablab, haseolus } \\
\text { sp., P. mungo, P. vulgaris, } \\
\text { P. aconitfolius, Vigna } \\
\text { unguiculata, V. vexillata, } \\
\text { Vigna sinensis }\end{array}$ & $\begin{array}{l}\text { TN, BR, MS, } \\
\text { HP, KA, KA, } \\
\text { RJ, AP, MP, GJ }\end{array}$ & $\begin{array}{l}\text { Butler \& Mcrae 1930, Jain } \\
\text { 1966, Joshi 1958, Nema \& } \\
\text { Mishra 1965, Patil 1966, } \\
\text { Prasad \& Sinha 1962, } \\
\text { Ramakrisnan \& Sundaram } \\
\text { 1955a, b, Sydow \& Butler } \\
\text { 1907a, b }\end{array}$ \\
\hline $\begin{array}{l}\text { U. eragrostidis } \\
\text { Tracy }\end{array}$ & $\begin{array}{l}\text { Eragrostis cynosuroides, } \\
\text { Eleusine coracana }\end{array}$ & BR, UP & $\begin{array}{l}\text { Dublish \& Singh 1977, Sydow } \\
\text { \& Butler 1907a }\end{array}$ \\
\hline $\begin{array}{l}\text { U. prominens } \\
\text { (DC.) Lév. }\end{array}$ & $\begin{array}{l}\text { Euphorbia hypericifolia, } \\
\text { E. hispida, E. } \\
\text { dracunculoides }\end{array}$ & $\mathrm{UP}, \mathrm{MS}$ & $\begin{array}{l}\text { Butlere \& Bisby 1931, Chavan } \\
\& \text { Bakare 1977, Cummins } \\
\text { 1943, Sydow \& Butler } 1938\end{array}$ \\
\hline $\begin{array}{l}\text { U. haussknechtii } \\
\text { Tranz. }\end{array}$ & Euphorbia pilosa & $\mathrm{J} \& \mathrm{~K}$ & Cummins 1943 \\
\hline $\begin{array}{l}\text { U. euphorbiae } \\
\text { Cooke \& Peck }\end{array}$ & Euphorbia thymifolia & GJ & Ajrekar 1912 \\
\hline $\begin{array}{l}\text { U. fritillariae } \\
\text { Thum. }\end{array}$ & Fritillaria roylei & $\mathrm{J} \& \mathrm{~K}$ & Arthur \& Cummins 1936 \\
\hline
\end{tabular}


Table 1 (continued)

\begin{tabular}{|c|c|c|c|}
\hline Taxa & Hosts & Distribution* & References \\
\hline $\begin{array}{l}\text { U. geranii (DC.) } \\
\text { G.H. Otth \& } \\
\text { Wartm. }\end{array}$ & $\begin{array}{l}\text { Geranium wallichianum, } \\
\text { G. aconitifolium }\end{array}$ & $\mathrm{J} \& \mathrm{~K}, \mathrm{BR}$ & $\begin{array}{l}\text { Cummins 1943, Sydow \& } \\
\text { Butler 1907a, Yadav } 1963\end{array}$ \\
\hline $\begin{array}{l}\text { U. sojae (P. Henn.) } \\
\text { Syd. \& P. Syd. }\end{array}$ & Glycine $\max$ & $\mathrm{TN}$ & Ramakrisnan $1951 b$ \\
\hline $\begin{array}{l}\text { U. hedysari- } \\
\text { obscuri (DC.) }\end{array}$ & $\begin{array}{l}\text { Hedysarum } \\
\text { cachemirianum }\end{array}$ & $\mathrm{J} \& \mathrm{~K}$ & $\begin{array}{l}\text { Arthur 1934, Arthur \& } \\
\text { Cummins } 1936\end{array}$ \\
\hline $\begin{array}{l}\text { Carestia \& Picc. } \\
\text { U. macintirianus } \\
\text { Barclay }\end{array}$ & Hemigraphis latebrosa & $\mathrm{HP}, \mathrm{BR}$ & Sydow \& Butler 1938 \\
\hline $\begin{array}{l}U . \text { heterogenus } \\
\text { Cooke }\end{array}$ & Hibiscus esculentus & MS & Uppal 1935 \\
\hline $\begin{array}{l}\text { U. muscari (Duby) } \\
\text { Graves }\end{array}$ & Hyacinthus orientalis & $\mathrm{TN}$ & Agarwal 1985 \\
\hline U. orientalis Syd. & $\begin{array}{l}\text { Indigofera linifolia, } I \text {. } \\
\text { cordifolia, I. glandulosa, } \\
\text { I. hirta }\end{array}$ & BR, MP, MS & $\begin{array}{l}\text { Sydow \& Butler 1907a, b, } \\
\text { Thite \& Patil } 1975\end{array}$ \\
\hline $\begin{array}{l}\text { U. indigoferae } \\
\text { Dietel \& Holw. }\end{array}$ & $\begin{array}{l}\text { Indigofera tinctoria, } \\
\text { I. linifolia }\end{array}$ & AP, MS & $\begin{array}{l}\text { Chavan \& Bakare 1977, Joshi } \\
\text { \& Reddy 1958, Joshi \& Reddy } \\
\text { 1959, Manoharachery } 1975, \\
\text { Saksena1955, Vasudeva } 1960\end{array}$ \\
\hline $\begin{array}{l}\text { U. gemmatus Berk. } \\
\text { \& M.A. Curtis }\end{array}$ & Jacquemontia paniculata & MS & Patil \& Thirumalachar 1981 \\
\hline U. hobsonii Vize & $\begin{array}{l}\text { Jasminum grandiflorum, } \\
\text { J. malbaricum, J. } \\
\text { scandens, J. auriculatum }\end{array}$ & $\begin{array}{l}\text { AP, J\&K, HP, } \\
\text { UK, KA, MS, } \\
\text { MP, BR, RJ }\end{array}$ & $\begin{array}{l}\text { Butler \& Mcrae 1930, Jain } \\
\text { 1966, Joshi 1958, Mishra } \\
\text { 1976, Parndekar 1964, Patil } \\
\text { 1966, Ramakrisnan \& } \\
\text { Sundaram 1955b, Rao 1989, } \\
\text { Sydow \& Butler 1907a, b, } \\
\text { Yadav \& Thirumalachar } 1955\end{array}$ \\
\hline $\begin{array}{l}\text { U. comedens } \mathrm{P} . \\
\text { Syd. \& Syd. }\end{array}$ & Jasminum sp. & AS & Vasudeva 1960 \\
\hline $\begin{array}{l}\text { U. pisi (DC.) G.H. } \\
\text { Otth }\end{array}$ & Lathyrus sativus & $\mathrm{BR}$ & Saksena 1956 \\
\hline $\begin{array}{l}\text { U. fabae (Pers.) de } \\
\text { Bary }\end{array}$ & $\begin{array}{l}\text { Lathyrus sphaericus, } L . \\
\text { sativus, } L . \text { odoratus, Lens } \\
\text { esculenta, Pisum sativum, } \\
P . \text { arvense,Vigna radiata, } \\
\text { Ocimum sp., Vicia faba, } \\
\text { V. biennis, V. hirsuta, V. } \\
\text { tetrasperma, V. } \\
\text { narborensis, V. gracilis, }\end{array}$ & $\begin{array}{l}\text { HP, UP, BR, } \\
\text { UK., MS, KA, } \\
\text { TN, MP }\end{array}$ & $\begin{array}{l}\text { Butler \& Mcrae 1930, Jain } \\
\text { 1966, Kapooria \& Sinha 1966, } \\
\text { Kulshreshtha 1998, Mishra } \\
\text { 1969, Mishra \& Khare 1969, } \\
\text { Mitter \& Tandon 1932, Patel } \\
\text { 1973, Pavgi \& Upadhyay } \\
\text { 1966, Sydow \& Butler 1938, } \\
\text { Sydow \& Butler 1907a, b, } \\
\text { Ramakrisnan \& Sundaram } \\
\text { 1955a, b }\end{array}$ \\
\hline $\begin{array}{l}\text { U. lespedezae- } \\
\text { procumbentis } \\
\text { (Schwein.) Lagerh. }\end{array}$ & Lespedeza bicolor & $\mathrm{J} \& \mathrm{~K}$ & Sydow \& Butler 1907a \\
\hline
\end{tabular}




\begin{tabular}{|c|c|c|c|}
\hline Taxa & Hosts & Distribution* & References \\
\hline U. rugulosus Pat. & Lespedeza eriocarpa & UK & Saksena 1956 \\
\hline $\begin{array}{l}\text { U. lespedezae- } \\
\text { sericae S. Ahmad }\end{array}$ & Lespedeza stenocarpa & UK & Sachin et al.1980 \\
\hline $\begin{array}{l}\text { U. behenis (DC.) } \\
\text { Unger }\end{array}$ & Lychnis indica & $\mathrm{J} \& \mathrm{~K}$ & Sydow \& Butler 1907a \\
\hline U. striatus Sch. & $\begin{array}{l}\text { Medicago sativa, } M \text {. } \\
\text { denticulata }\end{array}$ & $\begin{array}{l}\mathrm{J} \& \mathrm{~K}, \mathrm{MS}, \mathrm{HR} \\
\mathrm{PB}, \mathrm{BR},\end{array}$ & $\begin{array}{l}\text { Joshi 1958, Pandotra \& Sastry } \\
\text { 1969, Sydow \& Butler } 1938\end{array}$ \\
\hline $\begin{array}{l}\text { U. mucunae } \\
\text { Rabenh. }\end{array}$ & $\begin{array}{l}\text { Mucuna pruriens, } M . \\
\text { deeringiana, } \text { M. rajada, } \\
\text { M. cochinchinensis }\end{array}$ & $\begin{array}{l}\text { WB, BR, MS, } \\
\mathrm{KL}, \mathrm{AP}\end{array}$ & $\begin{array}{l}\text { Butler \& Mcrae 1930, Chavan } \\
\text { \& Patil 1972, Prakash \& Singh } \\
\text { 1976, Rabenhorst 1878, } \\
\text { Sydow \& Butler 1907a, b }\end{array}$ \\
\hline $\begin{array}{l}U . \text { ramacharii } \\
\text { Ravinder \& } \\
\text { Bhagyan. }\end{array}$ & Ocimum sp. & AP & $\begin{array}{l}\text { Bhagyanarayana \& Ravinder } \\
1994\end{array}$ \\
\hline $\begin{array}{l}\text { U. orthosiphonis } \\
\text { T.S. Ramakr \& } \\
\text { Shriniv. }\end{array}$ & Orthosiphon glabratus & $\mathrm{TN}$ & $\begin{array}{l}\text { Ramakrisnan \& Srinivasan } \\
1950\end{array}$ \\
\hline U. superfluens Syd. & Panicum antidotale & MP & \\
\hline $\begin{array}{l}\text { U. linearis Berk. \& } \\
\text { Broome }\end{array}$ & $\begin{array}{l}\text { Panicum miliare, } P \text {. } \\
\text { repens }\end{array}$ & $\begin{array}{l}\text { MS, BR, TN, } \\
\text { CG, KA, AP }\end{array}$ & $\begin{array}{l}\text { Butler \& Mcrae 1930, Sydow } \\
\text { \& Butler 1907b }\end{array}$ \\
\hline $\begin{array}{l}\text { U. dactylidis var. } \\
\text { poae (Rabenh.) } \\
\text { Grove }\end{array}$ & Роа аппиа & $\mathrm{HR}$ & Saini \& Chand 1984 \\
\hline $\begin{array}{l}\text { U. polygoni- } \\
\text { avicularis var. } \\
\text { polygoni-avicularis } \\
\text { (Pers.) P. Karst. }\end{array}$ & $\begin{array}{l}\text { Polygonum aviculare, } P . \\
\text { cogatum, } P . \\
\text { paronychioides }\end{array}$ & $\mathrm{J} \& \mathrm{~K}, \mathrm{HP}, \mathrm{J} \& \mathrm{~K}$ & $\begin{array}{l}\text { Cummins 1943,Sydow \& } \\
\text { Butler 1907b, Sydow } 1938\end{array}$ \\
\hline $\begin{array}{l}\text { U. pontederiae } \\
\text { W.R. Gerard }\end{array}$ & Pontederia cordata & AS & Agarwal \& Sarbhoy 1986 \\
\hline $\begin{array}{l}\text { U. pseudoarthriae } \\
\text { Cooke }\end{array}$ & Pseudarthria viscida & MS & $\begin{array}{l}\text { Chavan 1975, Patil \& } \\
\text { Thirumalachar } 1981\end{array}$ \\
\hline U. dolicholi Arthur & Rhynchosia minima & MS & Arthur 1934, Patel et al. 1949 \\
\hline $\begin{array}{l}\text { U. rottboelliae } \\
\text { Arthur }\end{array}$ & Rottboellia speciosa & UP, HP, J\&K & Sydow \& Butler 1938 \\
\hline $\begin{array}{l}\text { U. rumicis } \\
\text { (Schumach.) G. } \\
\text { Winter }\end{array}$ & $\begin{array}{l}\text { Rumex dentatus, } R . \\
\text { vescarius, } R . \text { dentatus }\end{array}$ & HR, MS, J\&K & $\begin{array}{l}\text { Behera \& Mukherji 1974, } \\
\text { Patel et al. 1949, Pandotra \& } \\
\text { Sastry } 1969\end{array}$ \\
\hline $\begin{array}{l}\text { U. scillarum } \\
\text { (Grev.) G. Wint. } \\
\text { U. indicus Pat. }\end{array}$ & $\begin{array}{l}\text { Scilla indica, } S \text {. } \\
\text { hyacinthine } \\
\text { Scirpus affinis }\end{array}$ & RJ, MS & $\begin{array}{l}\text { Chakrabarty } 1983 \text {, Prasad et al. } \\
1962 \\
\text { Butler \& Bisby } 1960\end{array}$ \\
\hline U. scirpi Burrill & Scirpus maritimus & PB & Arthur \& Cummins 1936 \\
\hline $\begin{array}{l}\text { U. poonensis W.D. } \\
\text { More \& Moni }\end{array}$ & Sesbania aegyptiaca & MS & More \& Moniz 1964 \\
\hline
\end{tabular}


Table 1 (continued)

\begin{tabular}{|c|c|c|c|}
\hline Taxa & Hosts & Distribution* & References \\
\hline $\begin{array}{l}\text { U. setariae-italicae } \\
\text { Yoshino }\end{array}$ & $\begin{array}{l}\text { Setaria italica, } S . \\
\text { verticillata, } V . \text { glauca, } \\
\text { Eriochola trypheron, } \\
\text { Cordia rothii }\end{array}$ & $\begin{array}{l}\text { MS, AP, Bihar, } \\
\text { Kerela, UK, } \\
\text { UP, MP, WB }\end{array}$ & $\begin{array}{l}\text { Butler \& Mcrae 1930, Sydow } \\
\& \text { Butler 1938, } \\
\text { Dublish \& Singh 1977, Mishra } \\
\text { et al. 1976, Narasimhan \& } \\
\text { Thirumalachar } 1964\end{array}$ \\
\hline $\begin{array}{l}\text { U. sommerfeltii } \\
\text { Hyl., Jorst. \& } \\
\text { Nannf. }\end{array}$ & Solidago virgaurea & $\mathrm{HP}$ & $\begin{array}{l}\text { Annonymus 1954, Barclay } \\
\text { 1890, Dietel } 1890\end{array}$ \\
\hline $\begin{array}{l}\text { U. tenuicutis } \\
\text { MacAlpine }\end{array}$ & Sporobolus diander & AS, UP, MS & $\begin{array}{l}\text { Butler \& Bisby 1931, Dube et } \\
\text { al. 1979b, Goswami \& Singh } \\
\text { 1973, Patil \& Date 1980a, }\end{array}$ \\
\hline $\begin{array}{l}\text { U. wellingtonica } \\
\text { T.S. Ramakr. \& K. } \\
\text { Ramakr. }\end{array}$ & Sporobolus indicus & $\mathrm{TN}$ & $\begin{array}{l}\text { Ramakrisnan \& Ramakrisnan } \\
1948\end{array}$ \\
\hline $\begin{array}{l}\text { U. mussooriensis } \\
\text { Syd. \& P. Syd. }\end{array}$ & Stipa sibirica & Uttk. & Sydow \& Butler 1938 \\
\hline U. strobilanthus & Strobilanthes & HP, UP, UK, & Mitter \& Tandon 1938, Mishra \\
\hline Barclay & dalhausianus & $\mathrm{MP}$ & 1969, Sydow \& Butler 1938 \\
\hline $\begin{array}{l}\text { U. triandrae T.S. } \\
\text { Ramakr. \& Shriniv. }\end{array}$ & Themeda triandra & $\mathrm{TN}$ & $\begin{array}{l}\text { Ramakrisnan \& Srinivasan } \\
1950\end{array}$ \\
\hline $\begin{array}{l}\text { U. trifolii }(\mathrm{R} . \\
\text { Hedw.) Lév. }\end{array}$ & $\begin{array}{l}\text { Trifolium pratense, } \\
\text { T. repens, } T . \text { resupinatum }\end{array}$ & HR, J\&K, PB & $\begin{array}{l}\text { Hooda \& Saini 1990, Padwick } \\
\& \text { Khan 1944, Sokhi et al. } \\
\text { 1985, Sydow \& Butler 1907b, }\end{array}$ \\
\hline U. minor J. Schrot. & Trifolium resupinatum & & Annonymous 1950 \\
\hline $\begin{array}{l}\text { U. anthyllidis } \\
\text { (Grev.) J. Schroet. }\end{array}$ & $\begin{array}{l}\text { Trigonella foenum- } \\
\text { graecum }\end{array}$ & MS, RJ, HP & $\begin{array}{l}\text { Joshi 1958, Payak 1962, } \\
\text { Sydow \& Butler } 1907\end{array}$ \\
\hline $\begin{array}{l}\text { U. tripogonicola } \\
\text { Payak \& Thirum }\end{array}$ & $\begin{array}{l}\text { Tripogon jacquemontii, } \\
\text { T. lisboa }\end{array}$ & MS & Patil \& Date 1980b \\
\hline $\begin{array}{l}\text { U. trichoneurae } \\
\text { Doidge }\end{array}$ & Tripogon lisboae & MS, & Kaul 1962, Patil 1966 \\
\hline $\begin{array}{l}\text { U. trogonellae } \\
\text { Pass. }\end{array}$ & Trogonella emodi & UK. & Sydow \& Mitter 1933 \\
\hline $\begin{array}{l}\text { U. valerianae } \\
\text { (Schumach.) Lév. }\end{array}$ & Valeriana wallichii & $\mathrm{J} \& \mathrm{~K}$ & Pandotra \& Sastry 1969 \\
\hline $\begin{array}{l}\text { U. valerianae- } \\
\text { wallichii Arthur \& } \\
\text { Cummins }\end{array}$ & $\begin{array}{l}\text { Valeriana wallichii, } \\
\text { V. leschenaultia }\end{array}$ & $\mathrm{HP}, \mathrm{UK}$ & $\begin{array}{l}\text { Arthur \& Cummins 1936, } \\
\text { Butler \& Bisby } 1931\end{array}$ \\
\hline $\begin{array}{l}\text { U. viciae-fabae } \\
\text { (Pers.) J. Schroet. }\end{array}$ & $\begin{array}{l}\text { Vicia sativa, V. hirsuta, } \\
\text { Lathyrus aphaca }\end{array}$ & UP & $\begin{array}{l}\text { Butler \& Bisby 1931, Dube et } \\
\text { al. } 1979 \text { a, Shrivastava } 1979\end{array}$ \\
\hline $\begin{array}{l}\text { U. phaseoli } \mathrm{G} \text {. } \\
\text { Winter }\end{array}$ & Vigna capensis & MS & Chavan \& Bakare 1974 \\
\hline U. vignae Barclay & Vigna vexillata & $\mathrm{HP}$ & Barclay A 1891 \\
\hline U. vossiae Barclay & Vossia speciosa & $\mathrm{HP}$ & Barclay 1890 \\
\hline $\begin{array}{l}\text { U. pianhyensis } \\
\text { Henn. }\end{array}$ & Wedelia urticaefolia & $\mathrm{TN}, \mathrm{KL}$ & $\begin{array}{l}\text { Hosagouder 1985, Padwick \& } \\
\text { Merh } 1943\end{array}$ \\
\hline
\end{tabular}


Table 1 (continued)

\begin{tabular}{llll}
\multicolumn{1}{c}{ Taxa } & \multicolumn{1}{c}{ Hosts } & Distribution* & \multicolumn{1}{c}{ References } \\
\hline $\begin{array}{l}\text { U. wedeliae- } \\
\text { biflorae Boedijn }\end{array}$ & Wedelia urticaefolia & MS & $\begin{array}{l}\text { Ramakrisnan \& Subramanian } \\
1951\end{array}$ \\
$\begin{array}{l}\text { U. coronatus } \\
\text { Miyabe \& Nishida }\end{array}$ & Zizania latifolia & MN & Nagachan \& Verma 1984 \\
$\begin{array}{l}\text { U. ignobilis (Syd. } \\
\text { \& P. Syd.) Arthur }\end{array}$ & -- & -- & Butler \& Bisby 1931 \\
$\begin{array}{l}\text { U. pegleriae } \\
\text {. }\end{array}$ & -- & PB & Sokhi et al. 1985 \\
\hline
\end{tabular}

*Jammu \& Kashmir (J\&K); Punjab (PB); Haryana (HR); Delhi; Uttrakhand (Uttk); Uttar Pradesh (UP); Rajasthan (RJ); Madhya Pradesh (MP); Gujarat (GJ); Bihar, (CG) Chhattisgarh, Tamil Nadu (TN); Andhra Pradesh (AP); Maharashtra (MS); Karnataka; Kerala; Assam; West Bengal (WB) and Manipur (MN).

\section{Acknowledgements}

Authors gratefully thank their respective organizations for providing laboratory and valuable support throughout the study.

\section{References}

Agarwal DK. 1985 - Uromyces muscari: a new record from India. Indian Phytopathology 54(2), 275.

Agarwal DK, Sarbhoy AK. 1986 - Uromyces potenderiae - a new record from India. Proceedings of National Academy of Sciences 56(B), 393-394.

Agarwal GP, Nema KG, Beliram R. 1959 - Fungi causing plant diseases at Jabalpur (M.P.)-I. Proceeding of National Academy of Sciences 29, 310-315.

Ajrekar Sl. 1912 - The caster rust (Melampsorella ricini de Toni). The Journal of the Bombay National History Society 21, 1092-1095.

Ajrekar Sl, Tonapy BR. 1923 - A note on the life history of Uromyces aloes (Cke.) Magn. Journal of Indian Botany Society 3, 267-269.

Anonymous 1950 - List of common names of Indian plant diseases. Indian Journal of Agricultural Sciences 20, 107-142.

Anonymous 1954 - Index of fungi. CMI 2(9), 165-181.

Arthur JC. 1934 - Manual of rusts in United States and Canada. Lafayatte, Indiana.

Arthur JC, Cummins GB. 1936 - Rusts of North Western Himalaya. Mycologia 25, 397-406.

Asthana RP. 1957 - Some observation on incidence of Uromyces cicer-arientini on Cicer arientinum. Nagpur Agriculture College Magazine 31, 20A-20B.

Barclay A. 1890 - Descriptive list of Uredineae occurring in neighborhood of Shimla (Western Himalaya). Journal of Asiatic Society of Bengal 56, 350-375.

Barclay A. 1891 - Additional Uredineae from the neighborhood of Shimla (Western Himalaya). Journal of Asiatic Society of Bengal 60, 211-230.

Behera N, Mukherji KG. 1974 - Fungi of Delhi XXV. Chlamydobasidia dasguptii n. sp. and Polyschema indica Behra, Mukhrejee and Sharma. Norwegian Journal of Botany 21, 1-3.

Bhagyanarayana G, Ravinder EJ. 1994 - A new species of Uromyces on Ocimum from India. Mycotaxon 50, 127-129.

Bhagyanarayana G, Ramachar P, Niranjan Rao K. 1987 - A new species of Uromyces on Atylosa from India. Mycotaxon 30, 193-194.

Bhahadur P, Singh U. 1967 - Possibility of new collateral hosts for the rust of gram. Science and Culture 53, 538-539. 
Bilgrami KS, Jamaluddin, Rizwi MA.1991 - Fungi of India: List and references. Today and Tomorrow's Printers \& Publishers, New Delhi, India.

Butler EJ, Bisby GR. 1931 - The Fungi of India. Imperial Council of Agricultural Research in India. Science Monograph, 1. XVIII.

Butler EJ, Mcrae W. 1930 - Reports of Imperial Mycologists, in Reports of Agricultural Research Institute \& College Pusa, 1907-1930.

Chakrabarty P. 1983 - Light blight disease of Nepenthes khasiana Hk.f. Indian Botanical Reporter 2, 59-61.

Chaudhari KCB, Singh AK. 1974 - Foot rot disease of sesame (Corticum rolfsii). Science and Culture 40, 115-116.

Chavan PB. 1975 - Critical notes on some rust fungi of Maharashtra, India. Maharashtra Vigyan Mandir Patrika 10, 23-26.

Chavan PB, Bakare VB. 1973a. - New rust from India. Maharashtra Vigyan Mandir Patrika 8, 3643.

Chavan PB, Bakare VB. 1973b - New rust from India. Maharashtra Vigyan Mandir Patrika 9, 132139.

Chavan PB, Bakare VB. 1974 - New rust from India. Indian Phytopathology Society 27, 266.

Chavan PB, Bakare VB. 1977 - Critical notes on some rust fungi of Western Maharashtra, India. Botanique 8, 137-140.

Chavan PB, Patil SK. 1972 - Studies on some rust from India. Sydowia 26, 277-281.

Cooke MC. 1874 - Blight on tea and cotton. Grevillea 3, 75-76.

Cooke MC. 1876 - Some Indian fungi. Grevillea 4, 114-118.

Cummins GB. 1943 - Uredinales from North West Himalayas. Mycologia 35, 446-458.

Cummins GB, Hiratsuka Y. 2003 - Illustrated genera of rust fungi. $3^{\text {rd }}$ edition. American Phytopathological Society, St Paul, Minnesota, USA. pp 175-176.

Dietel P. 1890 - Uredineen aus dem Himalaya. Hedwigia 29, 259-270.

Dube VP, Shobha T, Singhal VP, Charaya MU. 1979a - Additions to the host range of Puccinia conclusa and Uromyces viciae-fabae. National Academy of Scientific Letters 2, 432.

Dube VP, Charaya MU, Khurana S, Tyagi S. 1979b - New host records for two species of Uromyces. Indian Phytopatholgy 32, 154-155.

Dublish PK, Singh PN. 1977 - Phytopathogenic fungi of Meerut, some new records. Current Science 46, 168.

Goswami RN, Nagachan SV. 1979 - Uromyces pavgii Goswami \& Nagachan - a new rust taxon on Achyranthes aspera. Current Science 48, 697-698.

Goswami RN, Singh KL. 1973 - Uredinales of North East India. Indian Phytopathology 23, 310314.

Hooda I, Saini LC. 1990 - Occurrence of Uromyces trifolii (DC) Lev. on Trifolium resupinatum L. Crop Research 3(2), 302-305.

Hosagouder VB. 1985 - Teleomycetes of South India. India Phytopathology 38(2), 278-281.

Jain AC, Nikam BG, Kulkarni SN, Sharma OP. 1966 - Fungi of Gwalior and Indore region. The Vikram 4, 181-187.

Jamaluddin S, Goswami MG, Ojha BM. 2004 - Fungi of India (1989-2001). Scientific Publishers (India) Jodhpur.

Joshi NC. 1958. Fungi of Ajmer (Raj.)-III. Proceeding of National Academy of Sciences 28(B), 303-307.

Joshi LM, Reddy AR. 1958 - Taxonomic studies of Uromyces of Indigofera species in India. Indian Phytopathology 11, 59-61.

Joshi LM, Reddy AR. 1959 - Some observation on Uromyces indigoferae Diet. \& Holw. the rust of Indigofera linifolia. Indian Phytopathology 12, 25-28.

Kapooria RG, Sinha S. 1966 - Studies on the host rage of Uromyces fabae (Pers.) de Bary. Indian Phytopathology 19, 229-230. 
Kaul TN. 1962 - Occurrence of Gnomonia leptostyla (Fr.) Ces. \& de. Not. on wheat in India. Current Science 31, 349.

Khan MK. 1994 - New host records of some foliicolous fungi from India. Indian Phytopathology 47(3), 274-275.

Kulshreshtha M, Gulabani A, Agarwal DK, Sarbhoy AK. 1998 - Some new additions to fungi of India. Indian Phytopathology 51(1), 104.

Magdum DK. 1967 - Uromyces vestergreni Syd. from Malvan Maharashtra. Maharashtra Vigyan Mandir Patrika 2, 41-42.

Manoharachery C. 1975 - New host records of some interesting fungi from India. Science and Culture 41, 237.

Mishra RP. 1969 - The Uredineae of Jabalpur (M.P.)-II. Proceeding of Bihar Academy of Sciences $17,76-80$.

Mishra RP, Khare MN. 1969 - Screening of Lathyrus germplasm collection against rust Uromyces fabae (Pers.) de Bary. Journal of Applied Sciences 1, 54-55.

Mishra RP, Nema KG. 1976 - Uredineae of Jabalpur (M.P.)-III. JNKVV Research Journal 10, 132-135.

Mishra RP, Nema KG, Singh SS. 1976 - Uredineae of Jabalpur (M.P.)-III. JNKVV Research Journal 10, 136-139.

Mitter JH, Tandon RN. 1932 - Fungus flora of Allahabad. Journal of Indian Botanical Society 9, 190-198.

Mitter JH, Tandon RN. 1938 - Fungus of Nainital Part-II. Journal of Indian Botanical Society 17, 177-182.

More WD, Moniz L. 1964 - Telial stage of the rust on Sebania aegyptiaca Poir. Current Science $33,449$.

Mukherji KG, Juneja RC. 1974 - Fungi of India: Supplement to the list of Indian Fungi. Emkay Publications Delhi, India.

Nagachan SV, Verma ND. 1984 - Uromyces coronatus on Zizania latifolia. Indian Phytopathology 37,741 .

Narasimhan MJ, Thirumalachar ML. 1964 - Heteroecism in Uromyces setariae-italicae, the rust on Italian millet. Mycologia 56, 554-559.

Nema KG, Mishra RP. 1965 - The Uredineae of Jabalpur, M.P. Nagpur Agriculture College Magazine 6, 79.

Pachkheda AU, Vyawahare SV, Shreemali JL. 1985 - Two new species of Phoma from India. Current Science 54, 485-486.

Padwick GW, Khan A. 1944 - Notes on Indian Fungi-II. Mycological Papers 10, 17.

Padwick GW, Merh JL. 1943 - Notes on Indian Fungi-I. Mycological Papers 7, 7.

Pandotra VR. 1966 - Notes on fungi from Jammu and Kashmir -1. Proceedings of the Indian Academy of Sciences 54, 68-73.

Pandotra VR, Sastry KSM. 1969 - Fungi on medicinal and aromatic plants in the North-West Himalayas-V. Proceedings of the Indian Academy of Sciences - Section B 70 (2), 88-97.

Parndekar SA. 1964 - A contribution to the fungi of Maharashtra. Journal of University of Poona 26, 56-64.

Patel KS. 1973 - A Cercospora leaf spot of bajra (Pennisetum typhoides Stapf.) in Gujrat. Current Science 42, 32.

Patel MK, Kamat MN, Bhinde VP. 1949 - Fungi of Bombay, Supplement-I. Indian Phytopathology 2, 142-155.

Patil BV, Thirumalachar MJ. 1968 - Life history and heteroecium of Uromyces commelinae Cke. Indian Phytopathology 32, 324-330.

Patil BV, Thirumalachar MJ. 1981 - Some new and interesting rusts from Maharashtra, India. Sydowia 25, 149-156.

Patil SD. 1966 - Rust of Mahabaleshwar. The Proceeding of the Autmn School in Bombay Mahabaleshwar 254-261. 
Patil SD, Date KG. 1980a - New host records for Puccinia and Uromyces from Maharashtra. MVM Patrika 15, 81-82.

Patil SD, Date KG. 1980b - New records for Puccinia and Uromyces from Maharashtra. Maharashtra Vigyan Mandir Patrika 8, 32-35.

Pavgi MS, Upadhyay HP. 1966 - Parasitic fungi from North India-IV. Mycopathologia et Mycologia Applicata 30, 257-260.

Payak MM. 1949 - Some parasitic fungi collected in vicinity of Banaras. Indian Phytopathology 2, 190-193.

Payak MM. 1962 - Natural occurrence of gram rusty in uredial stage on Trigonella polycerata L. in Shimla hills. Current Science 31, 433-434.

Ponappa KM. 1969 - On five fungi associated with coconut palm in India. Indian Phytopathology $22,342-345$.

Prakash O. 1979 - A new mango disease and its cure. Indian Journal of Mycology and Plant Pathology 8, 32 .

Prakash O, Singh UN. 1976 - Basal rot of mango seedlings caused by Sclerotium rolfsii. Journal of Mycology and Plant Pathology 7, 75.

Prasad N, Sharma LC, Singh RD. 1962 - Two new rusts from Rajasthan. Indian Phytopathology $15,80-83$.

Prasad SS, Sinha BD. 1962 - Fungi causing diseases at Muzaffarour-I. Proceedings of National Academy of Sciences India 32(3), 435-438.

Rabenhorst L. 1878 - Fungi Europaei Exsiccati. Hedwigia 17, 31, 47, 59-63, 80-90, 171-176.

Ramachar P, Bhagyanarayana G, Kumar A. 1978. Additions to our knowledge of rusts (Uredinales) from Hydrabaad (India)-III. Proceedings of National Academy of Sciences India 87(B), 113118.

Ramakrisnan K, Subramanian CV. 1951 - The fungi of India-A second supplement. Journal of Madras University 21(B), 1-65.

Ramakrisnan TS. 1951a - Additions to fungi of Madras-XI. Proceedings of National Academy of Sciences India 34(B), 157-164.

Ramakrisnan TS. 1951b - Additions to fungi of Madras-XII. Proceedings of National Academy of Sciences India 35(B), 111-121.

Ramakrisnan TS, Rangaswamy G. 1948 - Uromyces acori Ramakrisnan \& Rangaswamy sp. nov. on Acorus calamus. Current Science 17, 240-41.

Ramakrisnan TS, Ramakrisnan K. 1948 - Additons to fungi of Madras-XII. Proceedings of National Academy of Sciences India 28(B), 50-70.

Ramakrisnan TS, Srinivasan KV. 1950 - On two new rust fungi. Current Science 19, 216.

Ramakrisnan TS, Srinivasan KV, Sundaram NV. 1952 - Additions to fungi of Madras-XIII. Proceedings of National Academy of Sciences India 36(B), 85-95.

Ramakrisnan TS, Sundaram NV. 1955a - Additons to fungi of Madras-XIII. Proceedings of National Academy of Sciences India 32(B), 58-64.

Ramakrisnan TS, Sundaram NV. 1955b - Additions to fungi of Madras-XVII. Proceedings of National Academy of Sciences India 41(B), 189-195.

Ramakrisnan TS, Srinivasan KV, Sundaram NV. 1953 - Additions to fungi of Madras-XIV. Proceedings of National Academy of Sciences India 37(B), 83-95.

Rao NG, Sikander A, Reddy MS. 1989 - Occurrence of severe rust on jasmine (Jasminum auriculatum) by Uromyces hobsoni in Andhra Pradesh. Journal of Research APAU 17(1), 76-78.

Rolla SR, Addala RS. 1963 - Uromyces commelinae Cooke new host plants. Current Science 29, 449-451.

Sachin SN, Sharma MR, Chatarji R. 1980 - Additions to the rust fungi of India. Indian Journal of Mycology and Plant Pathology 10, 130.

Saini LC, Chand JN. 1984 - Uromyces dactylidis on Poa annua. Indian Phytopathology 37, 586. 
Saksena HK. 1956 - Two new records of Uromyces sp. from India. Science and Culture 22, 337338.

Saksena SB. 1955 - Ecological factors governing the distribution of soil microfungi in some forest soils of Sagar. Journal of Indian Botanical Society 35, 262-298.

Sarbhoy AK, Agarwal DK, Varshney JL. 1975 - Fungi of India (1977-81). Associated Publishing Company New Delhi, India.

Sarbhoy AK, Varshney JL, Agarwal DK. 1980 - Fungi of India (1971-76). Navyug Traders Publishers, New Delhi, India.

Sokhi SS, Singh SJ, Singh BB. 1985 - Uredinae of Punjab State. Indian Journal of Plant Pathology 14(2), 160-162.

Srivastava RC. 1979 - Fungi causing plant diseases of Jaunpur UP-II. Indian Phytopathology 32, 289-290.

Sundaram NV. 1964 - Physiologic specialization in Uromyces leptodermisi Syd. Indian Journal of Agriculture Sciences 34, 215-218.

Sydow H. 1913 - Beitrage zur Kennitius der Pilze flora des Sudlichen Ostindiens. Annales Mycologici 11, 326-330.

Sydow H. 1938 - Fungi Himalayensis. Annales Mycologici 36, 437-442.

Sydow H, Butler EJ. 1907a - Fungi Indiae Orientalis-II. Annales Mycologici 5, 485-515.

Sydow H, Butler EJ. 1907b - Fungi Indiae Orientalis-IV. Annales Mycologici 10, 243-280.

Sydow H, Butler EJ. 1938 - Fungi Indiae Orientalis-I. Annales Mycologici 4, 424-445

Sydow H, Mitter JH. 1933, Fungi Indiae Orientalis-II. Annales Mycologici 43, 46-71.

Sydow H, Mitter JH, Tandon RN. 1937 - Fungi Indicae-III. Annales Mycologici 35, 222-243.

Thite AN, Patil MS. 1975 - Additions to the rust fungi of Maharashtra. Journals of Shivaji University, Kolhapur 15, 49-51.

Unger F. 1833 - Die Exantheme der Pflanzen und einige mit diesen verwandte Krankheiten der Gewächse: pathogenetisch und nosographisch dargestellt (in German). p. 277.

Uppal BN, Patel MK, Kamat MN. 1935 - The fungi of Bombay-VIII pp. 1-56.

Vasudeva RS. 1950 - Report of the head of the division of Mycology and Plant Pathology. Indian Council of Agricultural Research (ICAR), New Delhi, India, 1948-50.

Vasudeva RS. 1960 - The Fungi of India. Indian Council of Agricultural Research (ICAR), New Delhi, India.

Vidal-Russel R, Nickrent DL. 2008 - Evolutionary relationships in the showy mistletoe family (Loranthaceae). American Journal of Botany 95, 1015-1029.

www.indexfungorum.org -2016

www.speciesfungorum.org - 2016

Yadav AK. 2004 - Anticestodal activity of Trifolium repens extract. Pharmaceutical Biology 42, 656-658.

Yadav AS. 1963 - Contribution to the knowledge of Uredineae of Bihar-III. Indian Phytopathology $16,138-142$.

Yadav AS, Thirumalachar MJ. 1955 - A contribution to the knowledge of Uredineae of Bihar-I. Indian Phytopathology 8, 143-149. 